\title{
On the Rough Approximation of Non-Convex Set
}

\author{
Fangan Deng ${ }^{1}$ Tao Zhou $^{1}$ Yang $\mathrm{Xu}^{2}$ \\ ${ }^{1}$ Department of Mathematics, Shanxi University of Technology, Hanzhong 723001, China \\ ${ }^{2}$ Intelligent Control Development Center, Southwest Jiaotong University, Chengdu 610031, China
}

\begin{abstract}
In this article, we have established the concept of the upper and the lower approximation of nonconvex set. Meantime, the properties of rough approximation of non-convex set have been investigated. As we know, every non-convex set can be approached by the upper and lower approximation convex set with respect to a given direct. Finally, the relationship between the shadow of $u$ and $u$-direction of the rough approximation sets have been given.
\end{abstract}

Keywords: Convex set, Non-convex set, Rough approximation

\section{Introduction}

Let $V$ be a real linear vector space. Following we will introduce some basic definitions.

Definition 1 Let $S \subset V$. For every $\lambda \in(0,1)$, if

$$
\lambda x^{1}+(1-\lambda) x^{2} \in S,
$$

where $x^{1}, x^{2} \in S$, then $S$ is said to be convex in $V$.

Definition 2 Let $S \subset R^{m}$ be a non-empty set, $u \in$ $R^{m}$ be a non-zero vector. Then

(1) If $\lambda \in(0,1)$, and for every $x^{1}, x^{2} \in S$, there exist the real number $\gamma \geq 0$ such that

$$
\lambda x^{1}+(1-\lambda) x^{2}+\gamma u \in S,
$$

then $S$ is called a convex set with respect to $u$ direction;

(2) The Set

$$
S(u)=\{y-\lambda u \mid y \in S, \lambda \geq 0\}
$$

is called the u-shadow of $S$. The following diagram can best expressed (see fig. 1).

Remark 1 Any non-empty convex set $S \subset R^{m}$ is convex set with respect to u-direction, for any nonzero vector $u \in R^{m}$. Besides, for any non-empty set $S$, we obtain $S \subset S(u)$.
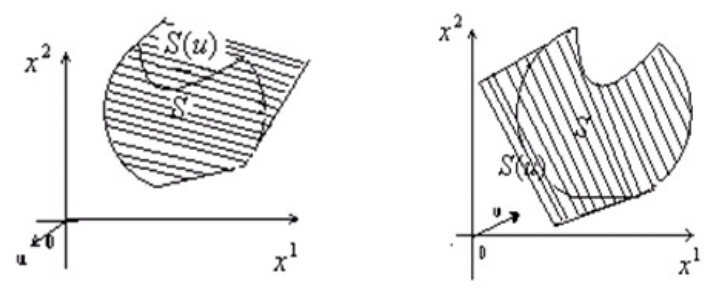

Fig. 1: u-shadow of S.

\section{Preliminaries}

As well known, convex sets are of good character, but not every set is convex. So how to express the non-convex set with a pair of rough approximation convex set is the main emphasis of our paper. For the sake of convenience, we will establish the following concept.

Definition 3 Let $S \subset V, \forall x^{1}, x^{2} \in S$. A direction can be established by the segment $\lambda x^{1}+\lambda x^{2}$, which denoted by $p$.

According to Definition 3, the non convex set $S$ can be divided into affirmative convex set and possibly convex set, with the aid of the line paralleled to $p$. If $S$ is a finite field, then there must exist a unique segment $S_{L}$. With this segment, a maximal convex subset can be confirmed, which is the maximal subset among the affirmative convex set, and we call it the generalized lower approximation set of $\mathrm{S}$ with respect to $p$ - direction, denoted by $\underline{S}_{p}$. The following form can better express

$$
\underline{S}_{p}=\cup\left\{Y \in V \backslash S_{L} \mid Y \subset S\right\} .
$$




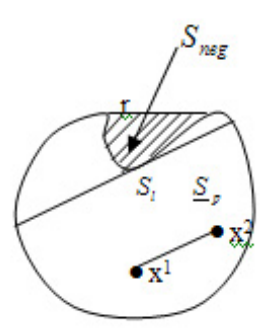

Fig. 2: Sketch map.

Clearly, $\forall x^{1}, x^{2} \in \underline{S}_{p}$, we obtain $\lambda x^{1}+(1-\lambda) x^{2} \in$ $\underline{S}_{p}$.

Definition 4 In definition 4, every direction $p$ is equivalent to a equivalence relation. In $S$, every segment paralleled to $p$ belongs to the same equivalence class.

It is worthy pointing that $S_{p}$ is not convex in general. If we add a region $S_{n e g}$ to $S$, and make $S \cup S_{n e g}$ become the minimal convex set including $S-S_{p}$, then $S \cup S_{n e g}$ is said to be a generalized upper approximation set of $S$ with respect to $p$ - direction, denoted by $\bar{S}_{p}$. The following form can better express

$$
\bar{S}_{p}=\cup\left\{Y \in V \backslash S_{L} \mid Y \cap S \neq \emptyset\right\}=\underline{S}_{p} \cup S_{b n},
$$

where $S_{b n}=\left\{S-S_{p}\right\} \cup S_{n e g}$. Clearly, $\underline{S}_{p} \subseteq S \subseteq \bar{S}_{p}$ (see fig.2). According to the above definition and Remark, we can also get the following result.

(1) Let $S \subset V$. If $p$ is different, then we can get the same generalized upper approximation set $\bar{S}_{p}$ and different lower approximation set $\underline{S}_{p}$;

(2) If $S$ is convex, then $\bar{S}_{p}=\underline{S}_{p}$.

Definition 5 [2] Let $S \subset V$. Then the intersection of all the convex sets including $S$ in $V$ is called convex hull, denoted by $\operatorname{co}(S)$. The convex hull is the smallest convex set including $S$.

\section{Main Results}

Theorem 1 Let $S \subseteq V$. Suppose that $p_{1}$ and $p_{2}$ are two directions, then the following results hold.

(1) If $p_{1} \| p_{2}$, then $\underline{S}_{p_{1}}=\underline{S}_{p_{2}}$;

(2) If $p_{1} \nVdash p_{2}$, then $\underline{S}_{p_{1}} \cap \underline{S}_{p_{1}} \neq \emptyset$, and $\underline{S}_{p_{1}} \cap \underline{S}_{p_{1}}$ is also convex.

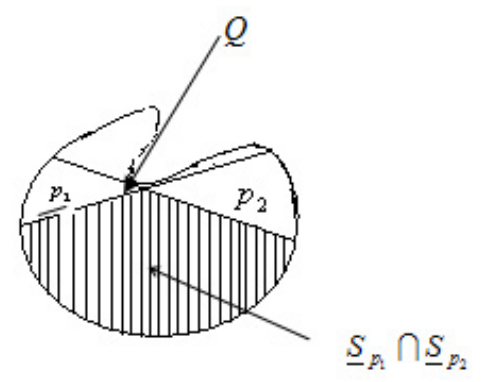

Fig. 3: $\underline{S}_{p_{1}} \cap \underline{S}_{p_{1}}$.

Proof.(1) By Definition 4, it can be easily to get the result.

(2) Let $l_{P_{1}}$ and $l_{p_{2}}$ be two polar line paralleled to $p_{1}$ and $p_{2}$, respectively, and $l_{p_{1}} \cap l_{p_{2}}=Q$. Then from the definition of polar line, we obtain $Q \in \underline{S}_{p_{1}}$. Similarly, we also get $Q \in \underline{S}_{p_{2}}$, that is, $\underline{S}_{p_{1}} \cap \underline{S}_{p_{1}} \neq$ $\emptyset . \forall y_{1}, y_{2} \in \underline{S}_{p_{1}} \cap \underline{S}_{p_{1}}$, then $\forall \lambda \in(0,1)$,

$$
y_{1}, y_{2} \in \underline{S}_{p_{1}} \Rightarrow \lambda y_{1}+\left(1-\lambda y_{2}\right) y_{2} \in \underline{S}_{p_{1}} .
$$

Similarly, we also have $\forall \lambda \in(0,1)$,

$$
y_{1}, y_{2} \in \underline{S}_{p_{2}} \Rightarrow \lambda y_{1}+\left(1-\lambda y_{2}\right) y_{2} \in \underline{S}_{p_{2}} .
$$

If there exist $\lambda_{1}^{*}, \lambda_{2}^{*}, \lambda_{1}^{*}+\lambda_{2}^{*} \in(0,1)$ satisfied

$$
\left(\lambda_{1}^{*}+\lambda_{2}^{*}\right) y_{1}+\left(1-\left(\lambda_{1}^{*}+\lambda_{2}^{*}\right)\right) y_{2} \notin \underline{S}_{p_{1}} \cap \underline{S}_{p_{1}},
$$

and take $\lambda_{1}^{*}+\lambda_{2}^{*}=\lambda^{*}$, then $\lambda^{*} y_{1}+\left(1-\lambda^{*}\right) y_{2} \notin$ $\underline{S}_{p_{1}} \cap \underline{S}_{p_{1}}$, that is,

$$
\begin{gathered}
\lambda^{*} y_{1}+\left(1-\lambda^{*}\right) y_{2} \in \underline{S}_{p_{1}} \text { and } \\
\lambda^{*} y_{1}+\left(1-\lambda^{*}\right) y_{2} \in \underline{S}_{p_{2}},
\end{gathered}
$$

which is a contradiction with the form (6) and (7). Hence $\forall \lambda_{1}, \lambda_{2}, \lambda_{1}+\lambda_{2} \in(0,1)$,

$$
\left(\lambda_{1}+\lambda_{2}\right) y_{1}+\left(1-\left(\lambda_{1}+\lambda_{2}\right)\right) y_{2} \in \underline{S}_{p_{1}} \cap \underline{S}_{p_{1}},
$$

therefore $\underline{S}_{p_{1}} \cap \underline{S}_{p_{1}}$ is also convex.

Theorem 2 Let $S \subseteq V$. Then the following statements hold.

(1) If $S$ is convex, then $\underline{S}_{p} \subset \operatorname{co}(S)=\bar{S}_{p}=S$, for any direction $p$;

(2) If $S$ is not convex, then $\underline{S}_{p} \subset \operatorname{co}(S)=\bar{S}_{p}$.

Proof. It is easy to get the result with the aid of Definition 3 and Definition 5

According to Theorem 2, for any $p, S$, we can see $\underline{S}_{p} \subseteq S \subseteq c o(S)=\bar{S}_{p}$. 


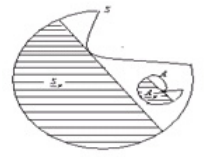

(a)

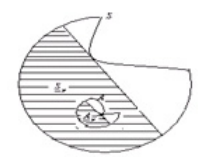

(b)

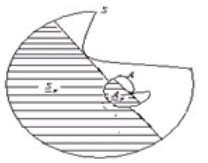

(c)
Fig. 4: Sketch map.

Theorem 3 Let $S_{1}, S_{2} \subseteq V$. Then

(1) $\bar{S}_{p}\left(S_{1} \cap S_{2}\right) \subset \bar{S}_{p} \cap \bar{S}_{p}\left(S_{2}\right)$;

(2) $\bar{S}_{p}\left(S_{1} \cup S_{2}\right) \subset \bar{S}_{p} \cup \bar{S}_{p}\left(S_{2}\right)$.

Theorem 4 Let $S \subseteq V$ be a non-convex set. Given a non-empty set $A \subseteq S$. Then

(1) If $A$ is not convex, and $\underline{S}_{p} \cap A \neq \emptyset$, then $\underline{S}_{p} \cap \underline{A}_{p} \neq \emptyset$

(2) If $A$ is not convex, and $A \subseteq \underline{S}_{p}$, then $\underline{A}_{p} \subseteq$ $\underline{S}_{p}, \underline{A}_{p} \subseteq \bar{S}_{p}$.

Proof. (1) By the fact that $A \subseteq S$ and $\underline{A}_{p} \subseteq$ $A$, then we have $\left(\underline{A}_{p} \cap \underline{S}_{p} \subseteq\left(A \cap \underline{S}_{p}\right)\right)$. Since $A \cap \underline{S}_{p}=$ $\emptyset, \underline{S}_{p} \cap \underline{A}_{p} \neq \emptyset$.

(2) Clearly, $\underline{A}_{p} \subseteq \underline{S}_{p}$ so following we will prove $\underline{A}_{p} \subseteq \bar{S}_{p}$. In fact, from the assumption that $A \subseteq \underline{S}_{p}$, we have $c o\left(A \cap \underline{S}_{p}\right)=c o(A)=c o(A) \cap \cos \underline{S}_{p}$. $)$ Besides, because $\underline{S}_{p}$ is convex, so $\operatorname{co}\left(\underline{S}_{p}\right)=\underline{S}_{p}$, hence $\underline{A}_{p}=c o(A) \subseteq \bar{S}_{p}$.

Meanwhile, according to the definition of $u-$ shadow of $S$ and the generalized rough approximation of $u$ - direction, we can also get the following result.

Proposition 1 (1) If $u$ - shadow of $S$ is convex, then $\bar{S}_{u} \subseteq S(u)$;

(2) If $u-$ shadow of $S$ is not convex, then $\bar{S}_{u} \cap$ $S(u)=S$

(3) $\underline{S}_{u} \subseteq S(u)$.

\section{Acknowledgments}

This work is supported by the Natural Science Foundation of China(No. 70472072, 60474022), and the Doctor Foundation of Shaanxi University of Technology. Besides, the authors wish to thank the referee for the valuable comments and suggestions which helped to improve the presentation of this note.

\section{References}

[1] B. Aupetit, Projections in real algebras, Bull. London Math. Soc., 13 (1981) 412-414.

[2] H. K. Du, X. Y. Yao, C. Y. Deng, Invertibility of linear combinations of two idempotents, Proc. Amer. Math. Soc., 134 (2005) 1451-1457.

[3] F.A. Deng, On the rough approximation of non-convex set, J.of University of Science and Technolongy of Suzhou(Natural Science), Vol.20, No.3 7-10. 